\title{
Mechanisms of Primary Axonal Damage in a Viral Model of Multiple Sclerosis
}

\author{
Jayasri Das Sarma, ${ }^{1}$ Lawrence C. Kenyon, ${ }^{2}$ Susan T. Hingley, ${ }^{3}$ and Kenneth S. Shindler ${ }^{4}$ \\ Departments of ${ }^{1}$ Neurology and ${ }^{2}$ Pathology, Anatomy, and Cell Biology, Thomas Jefferson University, Philadelphia, Pennsylvania 19107, ${ }^{3}$ Philadelphia \\ College of Osteopathic Medicine, Philadelphia, Pennsylvania 19131, and ${ }^{4}$ Scheie Eye Institute and FM Kirby Center for Molecular Ophthalmology, \\ University of Pennsylvania, Philadelphia, Pennsylvania 19104
}

\begin{abstract}
Multiple sclerosis (MS) is an inflammatory demyelinating disease of the CNS. Recent studies have demonstrated that significant axonal injury also occurs in MS patients and correlates with neurological dysfunction, but it is not known whether this neuronal damage is a primary disease process, or occurs only secondary to demyelination. In the current studies, neurotropic strains of mouse hepatitis virus (MHV) that induce meningitis, encephalitis, and demyelination in the CNS, an animal model of MS, were used to evaluate mechanisms of axonal injury. The pathogenic properties of genetically engineered isogenic spike protein recombinant demyelinating and nondemyelinating strains of MHV were compared. Studies demonstrate that a demyelinating strain of MHV causes concomitant axonal loss and macrophage-mediated demyelination. The mechanism of axonal loss and demyelination in MHV infection is dependent on successful transport of virus from gray matter to white matter using the MHV host attachment spike glycoprotein. Our data show that axonal loss and demyelination can be independent direct viral cytopathic events, and suggest that similar direct axonal damage may occur in MS. These results have important implications for the design of neuroprotective strategies for CNS demyelinating disease, and our model identifies the spike protein as a therapeutic target to prevent axonal transport of neurotropic viruses.
\end{abstract}

\section{Introduction}

The primary target in multiple sclerosis (MS) pathology is believed to be either myelin itself or the myelin-forming cell, the oligodendrocyte. Axonal damage described in early literature drew little attention because it was regarded as secondary to myelin injury (Kornek et al., 2000). Recently, there has been critical reappraisal of axonal damage in MS as gray matter involvement (Sharma et al., 2001) and axonal injury in white matter were reported (Lovas et al., 2000; Bjartmar et al., 2001). Axonal injury is likely irreversible and a major component of MS (Ferguson et al., 1997; Trapp et al., 1998, 1999), but it is not known whether axonal injury occurs exclusively secondary to myelin damage, or concomitantly with myelin damage from a direct neuronal insult. Mechanisms of axonal damage in MS are essentially unknown, although evidence suggests it may not be entirely immune mediated (Lucchinetti et al., 2001).

Axonal damage occurs in several viral infections, including human immunodeficiency virus (An et al., 1997; Raja et al., 1997), human T-lymphotropic virus (Iwasaki, 1990), and herpes simplex virus (Martin, 1984), as well as Theiler's murine enceph-

Received April 27, 2009; revised July 7, 2009; accepted July 9, 2009.

This work was supported by Research Grant RG3774A2/1 from the National Multiple Sclerosis Society, the M. E. Groff Surgical Medical Research and Education Charitable Trust (F76401), and Lindback Foundation Career Enhancement Award to J.D.S.; and National Institutes of Health Grant EY015098 and Career Development Award from Research to Prevent Blindness to K.S.S. We thank Elsa Aglow and Rhonda Walters for histological assistance and Ray Mead, Bio Imaging Core, University of Pennsylvania, for electron microscopic assistance.

Correspondence should be addressed to Jayasri Das Sarma at her present address: Department of Biological Sciences, Neuroscience Group, Indian Institute of Science Education and Research, Kolkata 700106, India. E-mail: dassarmaj@iiserkol.ac.in.

DOI:10.1523/JNEUROSCI.1975-09.2009

Copyright $\odot 2009$ Society for Neuroscience $\quad$ 0270-6474/09/2910272-09\$15.00/0 alomyelitis virus (TMEV) (Tsunoda and Fujinami, 2002; Tsunoda et al., 2003), whose infection has been associated with MS or animal models of MS. Some neurovirulent viruses, including herpes simplex, rabies, influenza and Borna viruses, can be anterogradely or retrogradely transported in axons (Kristensson, 1996; Mori and Kimura, 2001) leading to axonal degeneration. In TMEV infection, axonal injury is accompanied by oligodendrocyte apoptosis that precedes demyelination, implying that axonal injury can trigger demyelination. Viral-induced models of demyelinating disease therefore provide paradigms for advancing our understanding of the relationship between demyelination and axonal loss.

Infection of mice with neurotropic strains of mouse hepatitis virus (MHV) induces meningoencephalitis, followed by chronic inflammatory demyelination (Lavi et al., 1984). MHV was believed to primarily cause demyelination, with relative axonal preservation. However, some axonal damage was recently observed (Dandekar et al., 2001) in an adoptive-transfer model of neurovirulent strain MHV-JHM. We observed that a related strain (MHV-A59) induces axonal loss in optic nerve, in contrast to MHV-2, a nondemyelinating (NDM) MHV strain (Shindler et al., 2008). These observations raise the question of whether axonal loss induced by demyelinating (DM) strains could be a primary pathology rather than only secondary to demyelination, and molecular mechanisms mediating this axonal loss are not known. To further investigate mechanisms of axonal degeneration, we compared ultrastructural pathology of recombinant DM and NDM MHV strains.

Our studies reveal that infection with RSA59, a DM strain containing MHV-A59 recombined with its wild-type A59 spike gene, causes axonal loss concurrent with macrophage-mediated demy- 
A
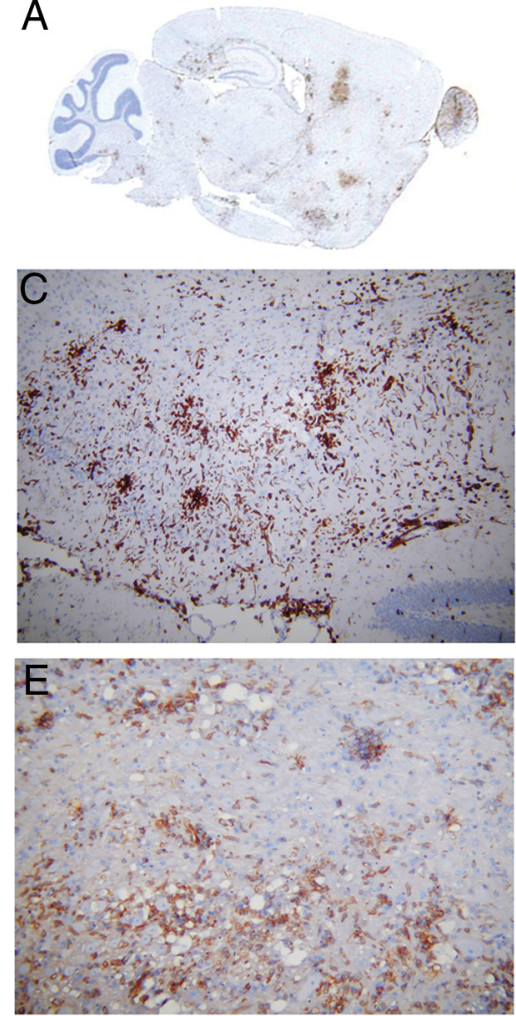

B
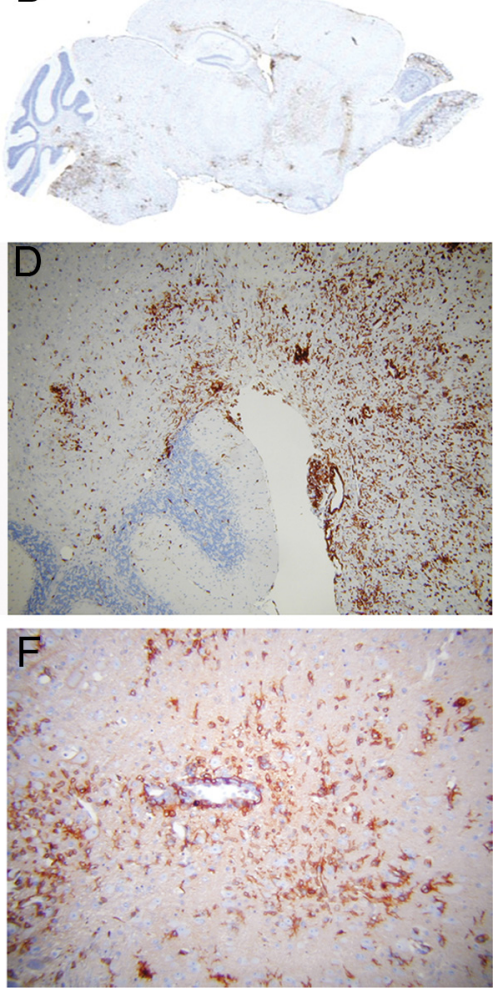

Figure 1. Characterization of inflammatory cells present during acute infection with RSA59 and RSMHV2 in mouse brain. Serial sagittal sections (5 $\mu \mathrm{m}$ thick) of brain from DM (RSA59) $(\boldsymbol{A}, \boldsymbol{C}, \boldsymbol{E})$ - and NDM (RSMHV2) $(\boldsymbol{B}, \boldsymbol{D}, \boldsymbol{F})$-infected mice at day 7 p.i were immunostained for $L C A(A-D)$ or $C D 11 b(E, F)$. LCA staining shows acute encephalitis in DM-infected mouse brain characterized by the presence of inflammatory infiltrates throughout the parenchyma in laser-scanned images $(\boldsymbol{A})$ and at high magnification $(\boldsymbol{C})$, with similar encephalitis in NDM-infected brain $(\boldsymbol{B}, \boldsymbol{D})$. The majority of $\mathrm{LCA}{ }^{+}$inflammatory cells stained positive for the macrophage/microglia marker CD11b in both DM (E)- and NDM $(\boldsymbol{F})$-infected mouse brain. $\boldsymbol{A}$ and $\boldsymbol{B}$ are laser-scanned images of glass slides. Original magnifications for $\boldsymbol{C}-\boldsymbol{F}$ are $100 \times$.

elination. In contrast, spinal cord infected with RSMHV2, a NDM strain containing the MHV2 spike gene in the MHV-A59 genome, shows only rare early axonal degeneration. Results suggest that mechanisms mediating both demyelination and axonal loss are dependent on spike-mediated axonal transport of viral particles from gray matter to white matter, and suggest axonal loss can occur due to direct viral attack in addition to, or rather than, occurring secondary to demyelination.

\section{Materials and Methods}

Viruses. Recombinant isogenic DM strain of MHV, RSA59, and NDM strain RSMHV2 were used from our previous studies (Das Sarma et al., 2002, 2008). RSA59 and RSMHV2 strains of MHV are isogenic (background is from demyelinating strain of MHV; MHV-A59) except for the spike gene, which encodes a glycoprotein expressed on the virion envelope that mediates many biological properties of MHV, including receptor attachment, fusion of viral and cell membranes during entry, cell-to-cell fusion during viral spread, and immune activation (Cavanaugh, 1995). These recombinant strains also express enhanced green fluorescence protein (EGFP).

Inoculation of mice. Four-week-old, MHV-free, C57BL/6 (B6) mice (Jackson Laboratory) were inoculated intracranially with $50 \% \mathrm{LD}_{50}$ dose of RSA59 strain (20,000 pfu) or RSMHV2 (100 pfu) as described previously (Das Sarma et al., 2002, 2008). Mice were monitored daily for mortality and signs of disease. Mock-infected controls were inoculated similarly but with an uninfected cell lysate at a comparable dilution. All experimental procedures were approved by the Institutional Animal Care and Use Committee.
Histopathological analysis. Mice were killed at the peak of inflammation (day 7) and/or day 30 postinoculation (p.i.), and were perfused transcardially with PBS followed by PBS containing $4 \%$ paraformaldehyde (PFA). Liver, brain, and spinal cord tissues were collected, postfixed in 4\% PFA overnight and embedded in paraffin. Brain and spinal cord tissues were sectioned at $5 \mu \mathrm{m}$ and stained with either hematoxylin/eosin (H\&E) for evaluation of inflammation, Luxol Fast Blue (LFB) to detect demyelination, or Bielchowsky silver impregnation (Czasch et al., 2006) for loss of axons. Experiments were repeated three times with 3-5 mice in each group. Areas of demyelination were quantified using a $0-3$ point scale as described earlier (Shindler et al., 2008), where $0=$ no demyelination; 1 = rare foci of demyelination; 2 = a few foci of demyelination; and $3=$ large (confluent) areas of demyelination. Briefly, two sections were examined from each of three spinal cord levels (cervical, thoracic and lumbar) for each mouse. All slides were coded and read in a blinded manner. Demyelination was scored based on detection of focal white matter areas lacking LFB staining using the relative three-point scale. Differences in degree of demyelination between groups were compared by Wilcoxon signed-rank test. To further quantify the level of demyelination at its peak $30 \mathrm{~d}$ postinoculation, identical sections of LFB-stained spinal cords from selected RSA59- and RSMHV2-infected mice were digitally scanned and analyzed on an Aperio Scanscope XT slide scanner and Aperio Imagescope version 10.0.36.1805 software (Aperio). A line was drawn surrounding all white matter regions on each slide, and the software was used to calculate the total area of white matter. Within the white matter, the combined area of foci that did not stain with LFB was also calculated, and were divided by the total white matter area to express the percentage of spinal cord white matter that was demyelinated.

To confirm expected virulence of the strains used, livers from the infected mice were embedded in paraffin, sectioned at $5 \mu \mathrm{m}$, and stained with H\&E (Das Sarma et al., 2000; Navas et al., 2001).

Immunohistochemical analysis. Serial sections from spinal cords were stained by the avidin-biotin-immunoperoxidase technique (Vector Laboratories) using 3, 3'-diaminobenzidine as substrate, and a 1:100 dilution of anti-CD45 (LCA; leukocyte common antigen, LY-5, BD PharMingen), anti-CD11b (OX42; Santa Cruz Biotechnology), or CD3 (PC3/188A; Santa Cruz) as primary antibodies. Control slides from mock-infected mice were incubated in parallel. To quantify the level of inflammation in spinal cord sections, LCA staining was scored by a blinded investigator as either absent (no staining), mild (few scattered LCA + cells), moderate (1-2 plaques of inflammatory cells), or severe (3 or more inflammatory plaques). Presence of moderate or severe inflammation was compared between groups by Fisher's exact test.

Ultrastructural studies. To further characterize the preservation of myelin and axons, mice were anesthetized, perfused with 4\% PFA, and spinal cords were harvested and fixed overnight in $2 \%$ glutaraldehyde. Samples for transmission electron microscopy (TEM) were postfixed with $1 \%$ osmium tetroxide, dehydrated, and flat embedded in Poly-Bed 812 epoxy resin (Polysciences). Half-micrometer-thick sections were cut from the lesional epicenter, stained with toluidine blue, and examined by light microscopy. Ultrathin TEM sections (600 $\AA$ ) were cut from representative foci of demyelination from the toluidine blue-stained semithin sections and mounted on 200 mesh copper grids, stained with uranyl acetate and bismuth subnitrite, and viewed under a JEOL JEM 1010 
electron microscope. Images were acquired with a Hamamatsu Orca-1 CCD camera and AMT Advantage Digital image Capturing software. Ultrathin sections were made from 5 mice from one cohort of RSA59- and RSMHV2infected mice.

Detection of viral antigen spread by EGFP. Cryostat sections were prepared from day 7 p.i. mice (Das Sarma et al., 2008). To detect EGFP fluorescence without immunostaining, brain and spinal cord cryostat sections were fixed in ice-cold 95\% ethanol for $20 \mathrm{~min}$, incubated in ice-cold PBS for $10 \mathrm{~min}$, and then mounted in Vectashield mounting medium (Vector Laboratories). Sections were visualized using an Olympus $\mathrm{X}-80$ microscope system with a $10 \times$ longworking distance UPlanF1 phase objective and a filter pack suitable for green fluorescence. Experiments were repeated in triplicate.

Isolation of primary neuronal cells. Primary hippocampal neurons were obtained from day 14 to day 16 embryonic C57BL/6 mice as previously reported (Pasick et al., 1994; Rall et al., 1997). Purity of isolated neuronal cells was determined by double-label immunofluorescence with neuronal marker anti-MAP2b and astrocyte marker anti-GFAP (glial fibrillary acidic protein).

Infection of primary neuronal cells with EGFP-tagged MHVs. On day 4 after explantation, neuronal cultures were infected at a multiplicity of infection (MOI) of 2:1 with RSA59 and RSMHV2 or mock infected with noninfected cell lysate. After allowing viral absorption for $1 \mathrm{~h}$, cells were washed and placed in fresh media without virus. At $0,24,36$, and $48 \mathrm{~h}$ after infection, cultures were examined by microscopy for EGFP fluorescence.

\section{Results}

\section{Comparative inflammatory CNS}

\section{pathology of DM versus NDM strains}

To characterize viral-induced CNS inflammation, mice were inoculated with RSA59 (DM) or RSMHV2 (NDM) and 7 d p.i. (peak inflammation) brain and spinal cord sections stained with $\mathrm{H} \& \mathrm{E}$ or LFB were examined. RSA59 induced meningitis, encephalomyelitis, hepatitis, and demyelination, whereas RSMHV2 caused only meningitis, encephalomyelitis, and hepatitis, but not demyelination, as shown previously (Das Sarma et al., 2000, 2008).

To characterize inflammatory cell types, brain sections from mice, day 7 p.i., were stained immunohistochemically with anti-CD45 (LCA; leukocyte common antigen), anti-CD11b (macrophage/microglial marker), or anti-CD3 (pan T-cell marker). The majority of inflammatory cells in either DM- or NDM-infected brains were immunoreactive for both LCA (Fig. $1 A-D$ ) and CD11b (Fig. $1 E, F$ ). Some CD3-stained infiltrating $T$ cells were also found (data not shown), although nonspecific background staining of neurons with available anti-CD3 antibodies made quantification difficult. Together, the data indicate that DM- and NDM-induced CNS inflam-
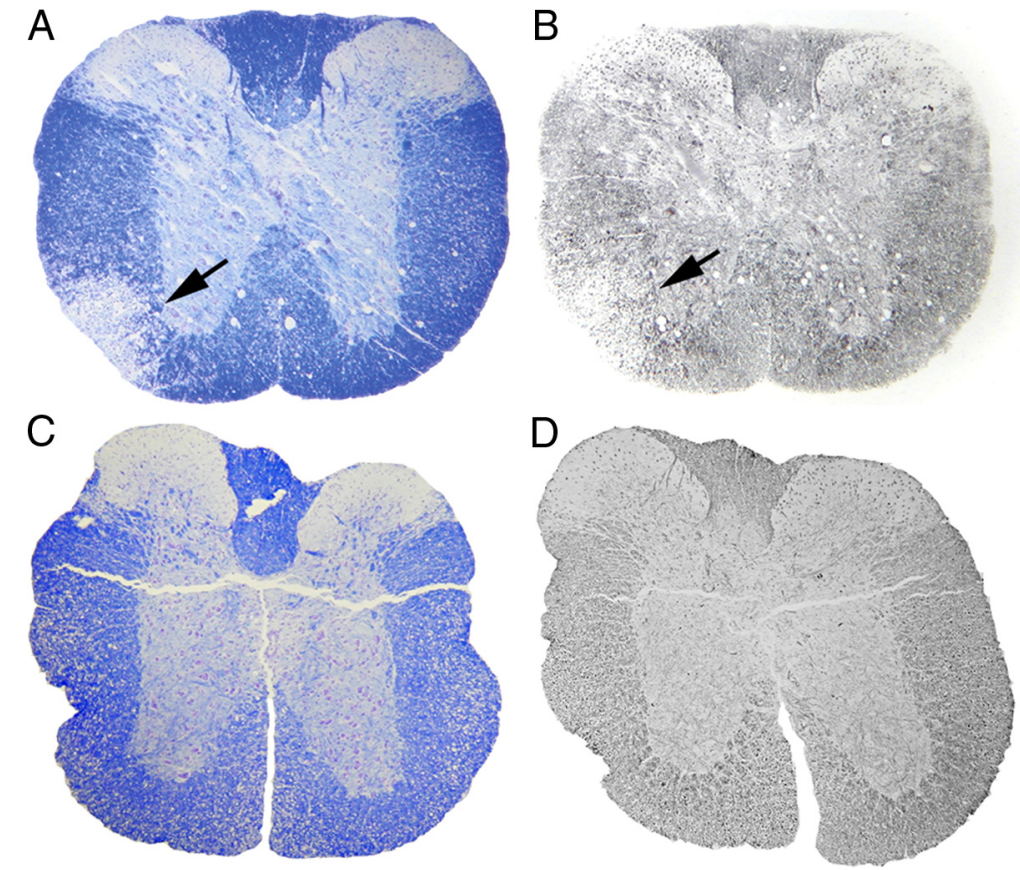

Figure 2. Demyelination and axonal loss in DM- and NDM-infected mouse spinal cord. Serial cross sections (5 $\mu \mathrm{m}$ thick) from DM- and NDM-infected mouse spinal cord at day 7 p.i. were stained for myelin with LFB $(A, C$ or by Bielchowsky silver impregnation $(\boldsymbol{B}, \boldsymbol{D})$. A, A large demyelinating plaque observed in DM-infected mouse spinal cord is shown (arrow indicates demyelinated area). $\boldsymbol{B}$, In an adjacent section, the same demyelinating plaque of DM-infected spinal cord showed loss of axons (arrow indicates area of axonal loss). C, Normal myelin was observed in NDM-infected mouse spinal cord. $\boldsymbol{D}$, No axonal loss was observed in the NDMinfected mouse spinal cord. Original magnification for $A-D$ is $40 \times$.
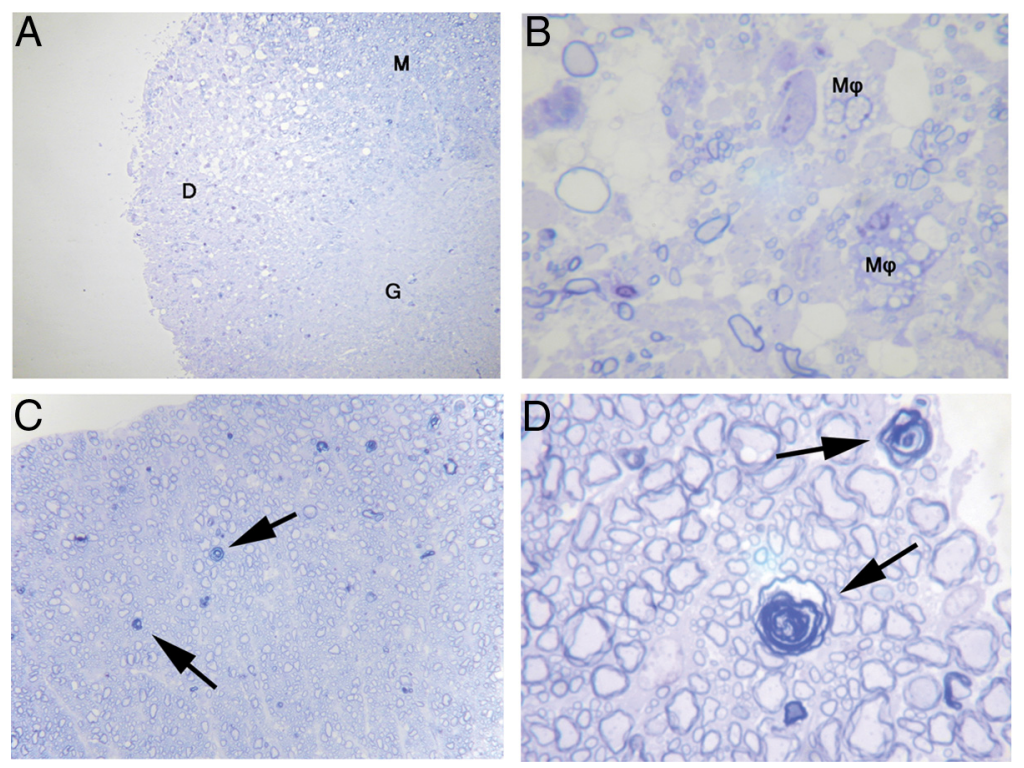

Figure 3. Toluidine blue-stained sections delineate preservation of myelin, axons, and axon-myelin coherence following DM and NDM infection. Spinal cord sections ( $1 \mu \mathrm{m}$ thick) of mice killed $30 \mathrm{~d}$ p.i. with DM and NDM were stained with toluidine blue. $\boldsymbol{A}$, $\boldsymbol{B}$, Large demyelinated plaques were observed in DM-infected mouse spinal cord. Myelinated spinal cord white matter region (M), demyelinated region of spinal cord white matter (D), gray matter $(G)$, and macrophages $(M \varphi)$ are marked. C, D, In NDM-infected mouse spinal cord, myelin remains relatively preserved with rare examples of early axonal degeneration characterized by loss of the central axon and collapse of the myelin sheath (arrows). Original magnification for $\boldsymbol{A}$ and $\boldsymbol{C}$ is $100 \times$ and for $\boldsymbol{B}$ and $\boldsymbol{D}$ is $1000 \times$. mation consists of a mixed population of inflammatory cells, predominantly macrophages/microglia as well as a smaller population of T lymphocytes.

Pathology was also assessed in cross sections from each spinal cord level at day 7 (peak inflammation) and day 30 (peak demy- 

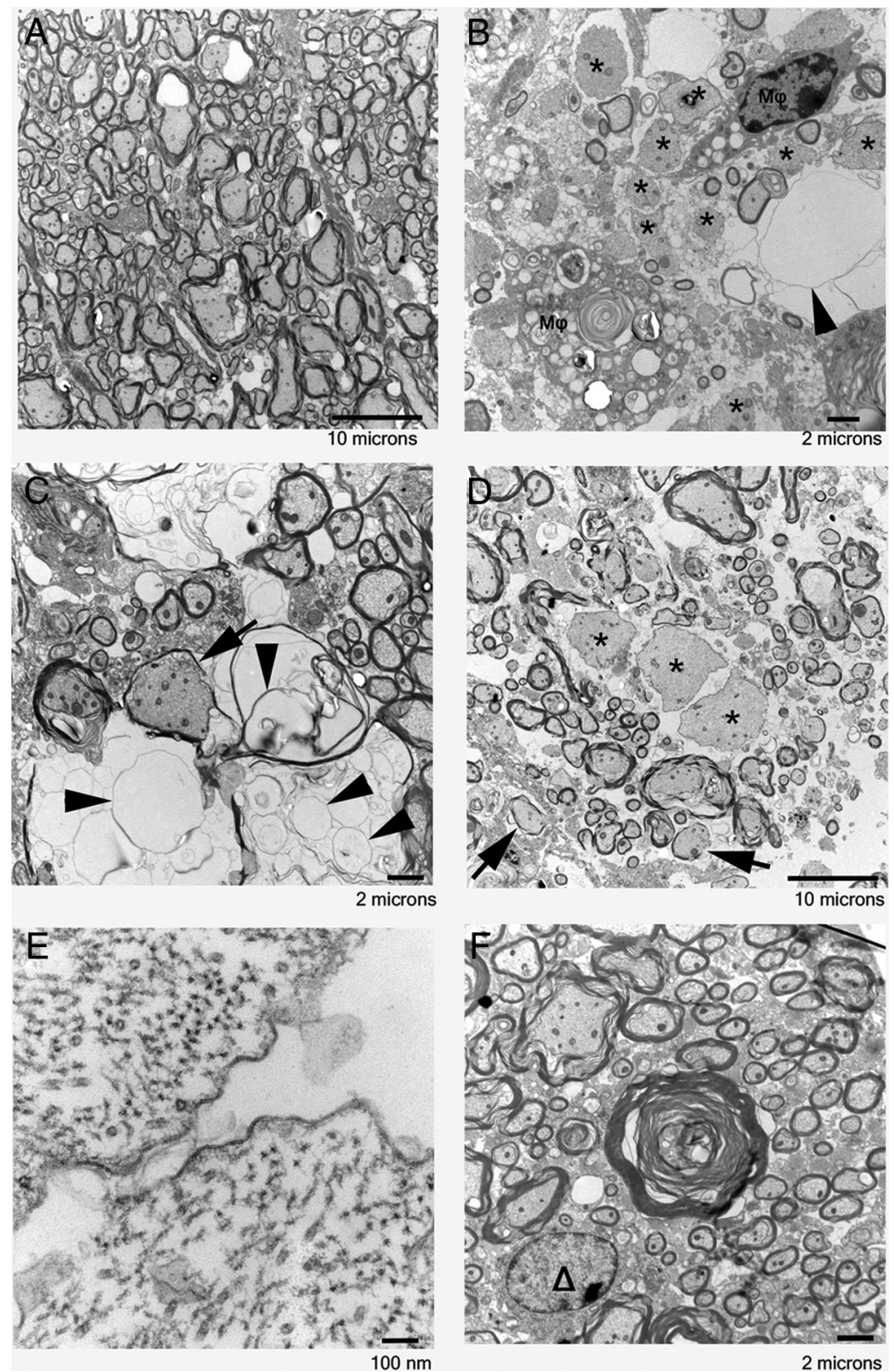

Figure 4. Electron micrographic analysis of demyelination and axonal loss in DM- and NDM-infected mouse spinal cord. Representative foci of demyelination and axonal injury were selected from the toluidine blue-stained sections shown in Figure 3 and processed for electron microscopy. $A$, Normal morphology of myelinated axons was exhibited from a nonplaque region adjacent to a demyelinated plaque of DM-infected mouse spinal cord. $\boldsymbol{B}-\boldsymbol{D}$, Demyelinating plaque in DM-infected mouse spinal cord showed extensive loss of myelin, hypomyelination (arrows), complete axonal degeneration with only residual empty vacuoles (arrowheads), naked axons with no myelin sheath at all (*), and macrophages (M $\varphi)$. $E$, A high-magnification image $(120,000 \times)$ of a naked axon surrounded only by a single lipid bilayer shows preservation of cytoskeletal elements (microtubules and intermediate filaments). $\boldsymbol{F}$, In contrast, NDM-infected mouse spinal cords show only rare examples of early axonal degeneration. This electron micrograph corresponds to a similar collapsed degenerating fiber seen in Figure 3D. $\Delta$ marks an oligodendrocyte nucleus. Such early axonal degeneration was not observed in mock-infected mice. Original magnifications: $\boldsymbol{A}, 3000 \times ; \boldsymbol{B}, 5000 \times ; \boldsymbol{C}, 6000 \times ; \boldsymbol{D}$, $3000 \times ; E, 120,000 \times$; and $F, 6000 \times$

elination) p.i. Similar to brain, both DM and NDM strains induced meningitis and myelitis (data not shown). Demyelinating plaques were detected by LFB stains for myelin (Das Sarma et al., 2008) at day 7 p.i. in DM-infected mice (Fig. 2A), and were quantified on a $0-3$ scale (Shindler et al., 2008). Day 30 p.i. tissue sections from DM-infected mice showed a similar pattern of demyelination as on day 7 , but the number and area of plaques was larger (see Fig. 7A). NDM-infected mice did not exhibit any demyelination either at day 7 (Fig. $2 C$ ) or at day 30 p.i. [average demyelination score/section $=0.0 ; n=9$ mice (54 sections) at day 7 and 10 mice (60 sections) at day 30 from 3 experiments]. Significant demyelination was detected in spinal cords from DM-infected mice at both day 7 [average demyelination score $0.81 \pm 0.52$ (mean \pm $\mathrm{SD}) ; n=9$ mice (54 sections); $p<0.0001$ vs NDM-infected mouse spinal cords] and day $30[1.88 \pm 0.68 ; n=14$ mice $(84$ sections); $p<0.0001]$. To further quantify the extent of demyelination, spinal cord sections from 3 NDM-infected and 3 DM-infected mice killed at day 30 p.i. were randomly selected for digital image analysis to measure the percentage of spinal cord white matter lacking LFB myelin staining. On average, spinal cord sections from DM-infected mice ( $n=18$ sections) had a significantly higher cumulative area of demyelination, covering $13.89 \pm$ $11.9 \%$ (mean $\pm \mathrm{SD}$ ) of all white matter regions, compared with NDM-infected mouse spinal cords that had no $(0.0 \%)$ detected demyelination ( $p=0.0003)$.

To avoid high mortality following NDM-induced hepatitis, we used $50 \%$ of the $\mathrm{LD}_{50}$ dose (100 pfu). In previous experiments, we ensured that the DM strain even at 5 and 50 pfu produced demyelination in $100 \%$ of infected mice; however, there was a reduction in the size and quantity of demyelinating plaques compared with a 50\% $\mathrm{LD}_{50}$ dose (Das Sarma et al., 2000).

Results show demyelination begins as early as day 7 p.i., indicating that DMinduced myelin damage begins during an early stage of acute inflammation, a finding not evident from earlier studies.

\section{Significant axonal loss occurs following RSA59 infection}

Though MHV-induced demyelination is characteristically described as sparing axons within areas of demyelination, we recently observed axonal loss with demyelination in the optic nerve of MHV-A59 (parental DM)-infected mice (Shindler et al., 2008). This led us to hypothesize that DM MHV may induce axonal loss in the spinal cord. To examine this, serial sections of spinal cords from RSA59 (DM)- and RSMHV2 (NDM)-infected mice were stained by Bielchowsky silver impregnation. DM-infected spinal cord sections at both day 7 and day 30 p.i. showed a significant decrease of axonal staining (Fig. 2 B). No axonal loss was evident in the NDM-infected mouse spinal cords either at day 7 (Fig. 2D) or at day 30 p.i. (data not shown).

To further evaluate the loss and/or preservation of myelin in DM and NDM-induced inflammatory plaques, semithin spinal cord sections cut at $1 \mu \mathrm{m}$ intervals from five infected mice at day 30 p.i. were stained with toluidine blue. Control mock-infected mouse spinal cord was used to evaluate for background fixation and/or postfixation artifacts. DM-infected spinal cords showed significant myelin loss within plaques (Fig. $3 A$ ). Moreover, high-magnification im- 
ages showed infiltrating macrophages filled with myelin debris (Fig. 3B). In contrast, in NDM strain RSMHV2-infected spinal cord sections, myelin is almost completely preserved, with only rare examples of early axonal degeneration, characterized by loss of the central axon and collapse of the myelin sheath (Fig. $3 C, D)$. The presence of this focal axonal degeneration was not detectable in prior studies of paraffin embedded tissues by silver impregnation.

High-resolution electron micrographic analysis confirms axonal loss concurrent with demyelination in RSA59-infected mouse spinal cord To further characterize axonal pathology at the ultrastructural level, representative foci of demyelination and axonal injury were selected from toluidine blue-stained sections, and $600 \AA$ ultrathin sections from Poly-Bed embedded blocks were processed for TEM. High-resolution TEM images show a combination of axonal degeneration and demyelination. There is extensive axonal loss and many residual empty vacuoles corresponding to totally degenerated fibers. In addition, there are numerous hypomyelinated fibers and naked axons without any myelin sheath, indicative of a demyelinating process (Fig. $4 B-D)$. These naked axons are fully intact with surrounding axolemmas and axoplasm with preserved microtubules and intermediate filaments (Fig. 4E). Importantly, for the first time, one mechanism of demyelination was revealed as macrophage-mediated myelin stripping. This is demonstrated in Figure 5 in which a macrophage is observed surrounding a myelinated axon. The myelin is unraveling, yet the axon is completely intact. At the inner border of the myelin sheath, multiple vacuoles are present as the entire myelin sheath is lifting off the axon. The macrophage cell membrane is in intimate contact with the outer portion of the myelin sheath as the macrophage strips away and engulfs the myelin sheath. Multiple vacuoles with myelin fragments are seen within the cytoplasm of the macrophage. In contrast, NDM-infected mouse spinal cords demonstrate no appreciable axonal loss and no features of demyelination. Specifically, there are no hypomyelinated fibers, no naked axons, no macrophages, and no evidence of macrophage-mediated myelin stripping. Instead, there are only rare examples of early axonal degeneration characterized by loss of the central axon and collapse of the myelin sheath (Fig. $4 F$ ) as previously seen in toluidine blue-stained sections (Fig. 3D).
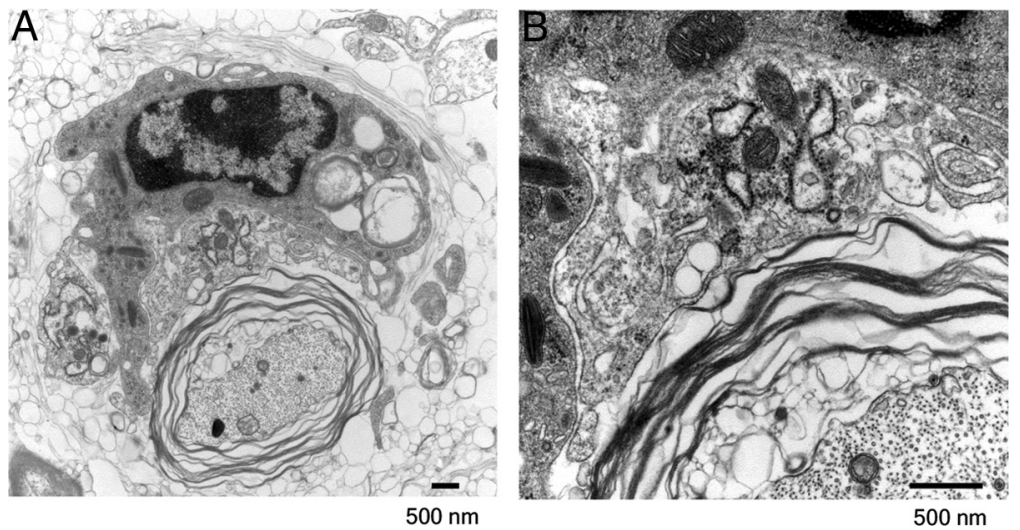

Figure 5. Macrophage-mediated myelin stripping in DM-induced demyelinating plaque. $A$, A macrophage (upper portion of figure) is observed surrounding an intact axon (lower portion of figure) with uncompacted myelin. Myelin figures are observed within the cytoplasm of this macrophage indicative of prior engulfment of myelin. $\boldsymbol{B}$, Higher magnification demonstrates close apposition of the macrophage cell membrane and an outer layer of uncompacted myelin. The axoplasm and axolemma are intact and the adjacent myelin shows vesiculation indicative of myelin degeneration. Original magnification for $A$ is $15,000 \times$ and for $B$ is $40,000 \times$.
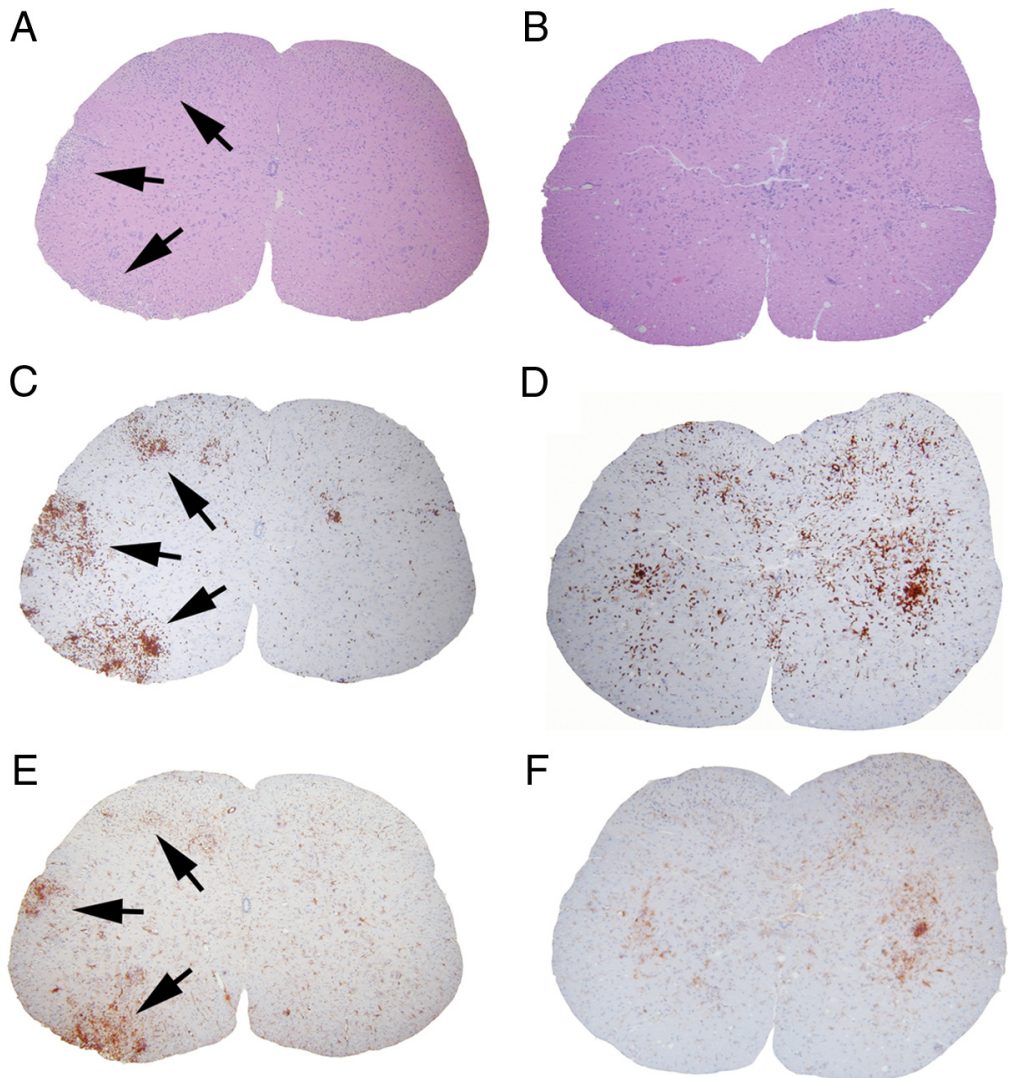

Figure 6. Distribution of inflammatory cells in the gray matter versus white matter in DM-and NDM-infected mouse spinal cord during acute infection. Serial cross sections ( $5 \mu \mathrm{m}$ thick) from DM- and NDM-infected mouse spinal cords at day $7 \mathrm{p}$.i. were stained with $\mathrm{H} \& \mathrm{E}(\boldsymbol{A}, \boldsymbol{B}), \mathrm{LCA}(\boldsymbol{C}, \boldsymbol{D})$, or CD11b $(\boldsymbol{E}, \boldsymbol{F})$. Several inflammatory lesions $(\boldsymbol{A})$ with infiltrating $\mathrm{LCA}^{+}$cells $(\boldsymbol{C})$ were observed in RSA59-infected mouse spinal cord (arrows indicate lesional areas in white matter). The majority of the LCA ${ }^{+}$inflammatory cells in the inflammatory plaques were positive for the microglia/macrophage marker CD11b (E). CD11b ${ }^{+}$cells in DM-infected mice are predominantly in the white matter. In NDM-infected mice, no discrete inflammatory lesions were observed (B); however, $\mathrm{LCA}^{+}$cells were observed (D) scattered throughout the gray matter. These cells were also strongly immunoreactive for CD11b $(\boldsymbol{F})$. Most of the LCA/CD11bimmunoreactive cells were restricted to gray matter with very little invasion of white matter. Original magnification for $\boldsymbol{A}-\boldsymbol{F}$ is $40 \times$.

\section{Spinal cord inflammatory plaques contain predominantly macrophages}

To characterize inflammatory cells within spinal cord plaques, serial sections from DM- and NDM-infected mice were stained 

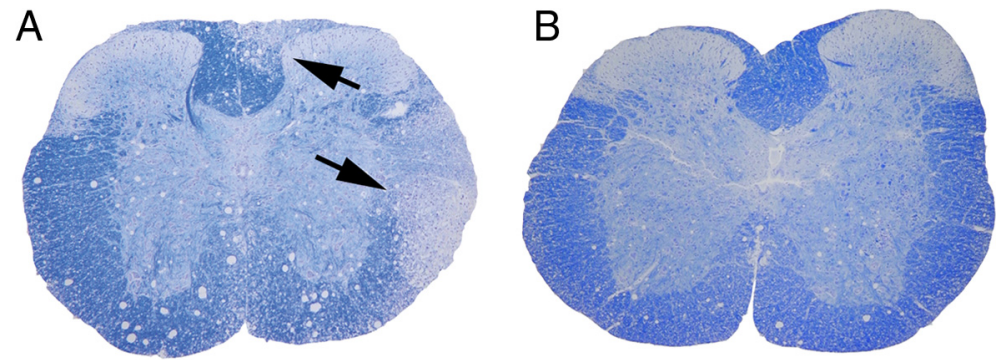

C

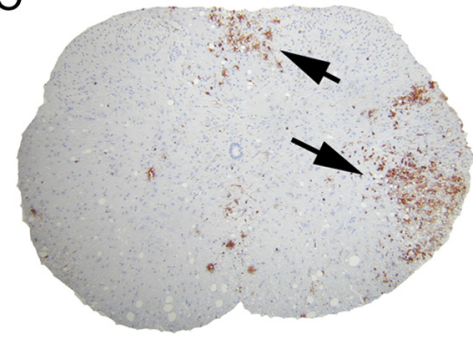

D

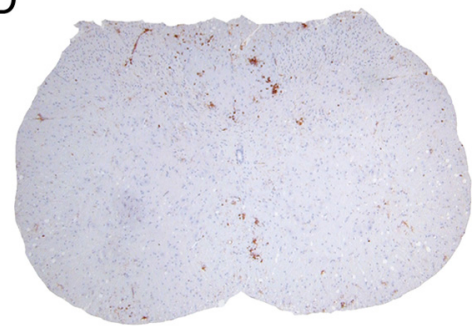

Figure 7. Distribution of inflammatory cells in the gray matter versus white matter in DM- and NDM-infected mouse spinal cords during chronic infection. Serial cross sections (5 $\mu \mathrm{m}$ thick) from DM- or NDM-infected mouse spinal cords at day 30 p.i. were stained with $\mathrm{LFB}(\boldsymbol{A}, \boldsymbol{B})$ or LCA $(\boldsymbol{C}, \boldsymbol{D})$. Large demyelinating lesions $(\boldsymbol{A})$ with infiltrating $\mathrm{LCA}^{+}$cells $(\boldsymbol{C})$ were observed in DM-infected mouse spinal cord white matter (arrows indicate lesion area) whereas normal myelin $(\boldsymbol{B})$ was observed in NDM-infected mouse spinal cord with only rare scattered LCA ${ }^{+}$cells in the gray matter $(\boldsymbol{D})$. Original magnification, $40 \times$.

with anti-CD45 (LCA), anti-CD11b, or anti-CD3. LCA staining confirmed the presence of infiltrating inflammatory cells (Fig. $6 C, D)$. Interestingly, the distribution of $\mathrm{LCA}^{+}$cells was significantly different after infection by the two viral strains. At day 7 p.i., the majority of infected cells in RSA59-infected spinal cord were localized to white matter with only occasional infiltration of gray matter (Fig. 6C), whereas in RSMHV2-infected spinal cord, $\mathrm{LCA}^{+}$cells were mainly restricted to gray matter (Fig. 6D). CD11b staining demonstrated that the majority of $\mathrm{LCA}^{+}$cells in the spinal cord were $\mathrm{CD}_{11 \mathrm{~b}}{ }^{+}$(Fig. 6E,F), similar to brain (Fig. $1 E, F)$. Some CD3-stained infiltrating $\mathrm{T}$ cells were also present (data not shown), although quantification of CD3-immunoreactive cells was limited by nonspecific background staining similar to brain. At day 30 p.i., $\mathrm{LCA}^{+}$cells were still present in abundance in demyelinating plaques produced following DM infection (Fig. $7 A, C)$ but in NDM-infected mice, no demyelinating plaques were present and very few $\mathrm{LCA}^{+}$cells were retained in the gray matter (Fig. $7 B, D$ ). Together, immunohistochemical data demonstrate that in DM- and NDM-strain-infected spinal cord there is an increase of $\mathrm{LCA}^{+} /$

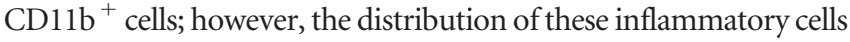
is significantly different between the two strains.

\section{DM and NDM strains differ in neuronal intracellular distribution of viral antigen}

To determine whether differential localization of viral antigen is responsible for the observed distribution of inflammatory cells, cross sections from spinal cord of DM- and NDM-infected mice were examined. Since the DM and NDM viruses used in these studies express EGFP, viral distribution was assessed directly by fluorescence microscopy. On day 7 p.i., the DM fluorescence was mainly restricted to white matter (Fig. $8 A, B$ ) with limited involvement of gray matter, whereas NDM fluorescence was restricted to gray matter (Fig. $8 C, D$ ). High-magnification fluorescence images in brain from day 7 p.i. demonstrate numerous viral antigen-positive neurons and their axons following DM infection (Fig. 8E), whereas far fewer fluorescent neurons and axons are observed following NDM infection (Fig. 8F).
The ability of DM and NDM to replicate and spread in vitro was compared using primary hippocampal neuronal cultures. Immunostaining of MAP2b and GFAP demonstrated that cultures consisted primarily of neurons with $<5 \%$ of cells exhibiting GFAP immunoreactivity (data not shown). At $12 \mathrm{~h}$ intervals, from 0 to $72 \mathrm{~h}$ p.i., cultures were monitored for viral antigen spread by fluorescence microscopy. At 24 h p.i. several infected cells were observed in DM-infected cultures (Fig. 8G), whereas, at $72 \mathrm{~h}$ p.i., the number of EGFP-positive cells was significantly increased (Fig. $8 H$ ). In contrast, NDMinfected cultures had few discrete cells positive for EGFP at $24 \mathrm{~h}$ p.i. (data not shown), and at $72 \mathrm{~h}$ p.i. the viral infection remained restricted only to the initially infected neurons (Fig. 8I), with little or no spread to adjacent cells, consistent with in vivo data.

\section{Discussion}

Results of the current studies provide important insights into the mechanisms of demyelination and axonal damage in CNS demyelinating diseases. Such insights are critical for designing rational therapeutic strategies for MS and related diseases. Specifically, our results demonstrate that axonal damage can occur concurrently with and independently of demyelination. This suggests that direct viral-mediated axonal damage can occur as a primary pathology, separate from the pathology of demyelination. Indeed, even the NDM strain of MHV used here lead to early, localized neurodegenerative changes, although complete loss of axons only occurred following infection with DM virus.

Our previous studies of MHV-induced meningoencephalomyelitis suggested that differential neural cell tropism may contribute to neurovirulence and induction of demyelination. Both DM and NDM strains infect neurons; however, these strains differ in their capacity to translocate to white matter as determined by fluorescence of EGFP-tagged viral particles. The current experiments indicate that axonal transport of viral particles is an important mechanism mediating not only the extent of axonal damage, but also the subsequent induction of demyelination in the spinal cord. DM strain shows white matter involvement early (by day 7 p.i.) and at later time points gray matter involvement is essentially absent in the spinal cord, with only minimal involvement in the brain. This observation further supports our earlier findings that DM and NDM strains differ in their viral antigen distribution during acute infection in the spinal cord (Das Sarma et al., 2008). Evaluation of axonal loss and demyelination in the spinal cord, where there is clear separation of gray matter and large tracts of white matter, demonstrated that DM MHV infection begins in the neuronal cell body and propagates centripetally to the axon (Fig. $8 E-I$ ), which subsequently induces axonal degeneration and demyelination. NDM strains were unable to propagate from gray to white matter and as a result, are unable to induce demyelination.

The migration and activation of numerous CD11b+ macrophages/microglia to the white matter following viral spread of DM MHV suggests that recruitment of these cells mediates demyelination. During DM strain chronic infection at day 30 p.i., 

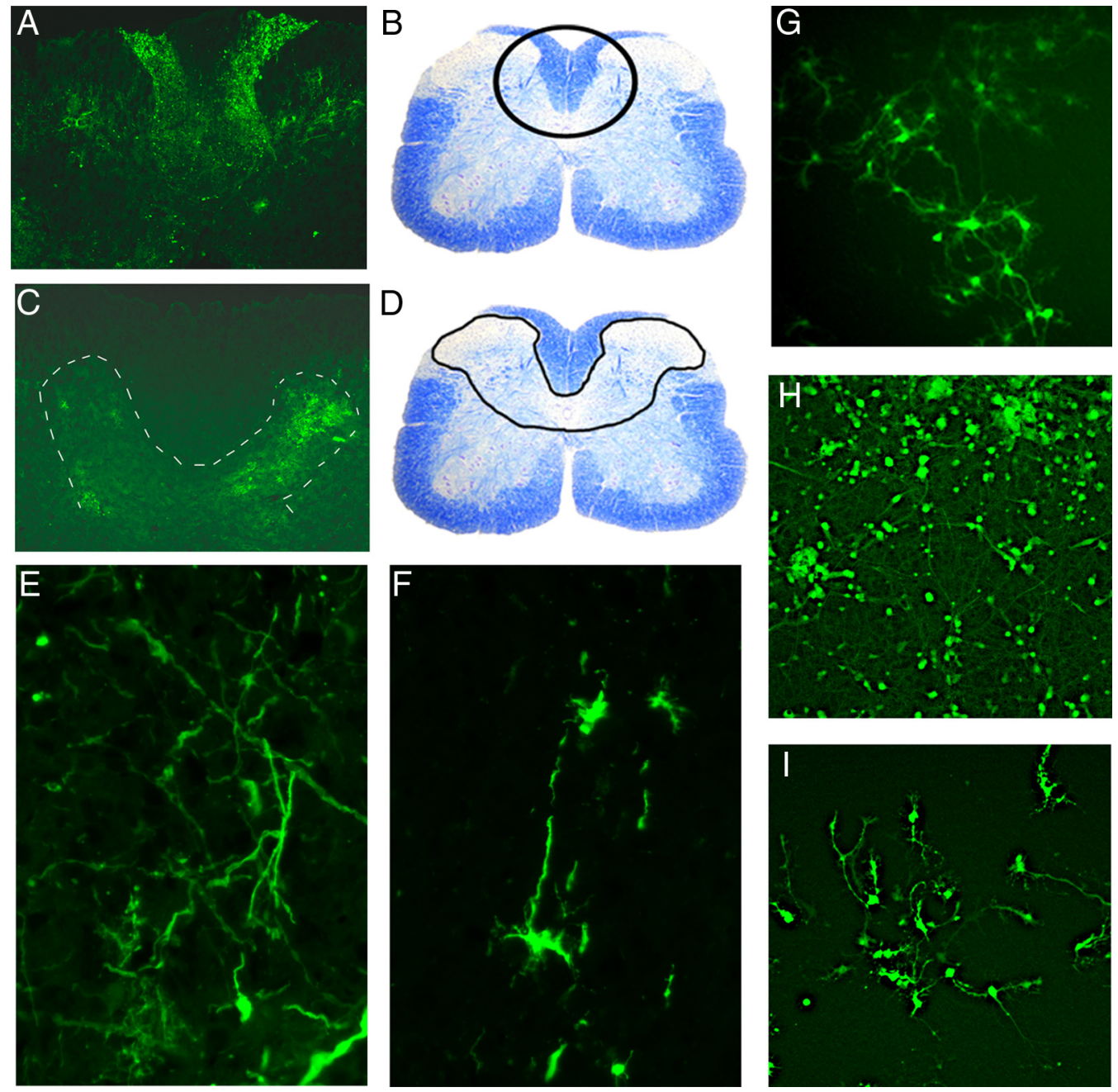

Figure 8. DM and NDM differ in their ability to translocate from gray matter to white matter during acute infection. DM- and NDM-infected mice were killed at day 7 p.i.; brain and spinal cord tissues were harvested and processed for frozen sections. Cryostat sections were postfixed with 95\% ice-cold ethanol for $20 \mathrm{~min}$ and observed by fluorescent microscopy for viral antigen (EGFP fluorescence). $\boldsymbol{A}$, In DM-infected spinal cord, viral antigen-positive EGFP expressing cells are present in both gray and white matter. $\boldsymbol{B}$, Area of EGFP-positive cells from $\boldsymbol{A}$ is marked in a corresponding LFB-stained section. C, In NDM-infected mice, EGFP-positive cells with viral antigen are predominantly restricted to the gray matter. D, Area of EGFP-positive cells from Cis marked in corresponding LFB-stained section. $\boldsymbol{E}$, Axonal distribution of the EGFP fluorescent viral antigen is evident in DM-infected brain. $\boldsymbol{F}$, NDM-infected neurons in the brain show considerably reduced axonal transport of viral antigen. G-I, To further confirm the ability of DM and NDM to infect neurons and spread from neuron to neuron, 4-d-old primary hippocampal neuronal cultures were infected with DM and NDM at MOI of 2. Representative fields containing an equivalent density of cells are shown in each image. G, At $24 \mathrm{~h} \mathrm{p.i.,} \mathrm{DM} \mathrm{infects} \mathrm{a} \mathrm{small} \mathrm{percentage} \mathrm{of} \mathrm{the} \mathrm{neurons} \mathrm{in} \mathrm{culture.} \boldsymbol{H}$, By $72 \mathrm{~h} \mathrm{p.i.,} \mathrm{DM-infected}$ neurons were observed to spread from one neuron to the next, as nearly all cells in the culture contain viral antigen despite initial infection of only a small percentage of cells. $I$, NDM-infected neurons on the other hand, demonstrate limited axonal transport efficiency at $72 \mathrm{~h}$ p.i., with only a small number of cells containing a bright concentrated signal that is retained after primary infection.

macrophages/microglia were still present within areas of demyelination, whereas macrophages/microglia and viral antigen were cleared from the gray matter following NDM strain infection. One plausible explanation is that MHV spreads intra-axonally in an anterograde manner within gray matter and when it reaches the white matter, viral particles are able to infect oligodendrocytes via direct cell-cell contact. A less likely possibility is that infection proceeds indirectly from neurons to oligodendrocytes by intermediary cells such astrocytes, microglia, or endothelial cells. Regardless of the mechanism of viral spread, ultimately, macrophages and microglia are recruited to the areas of infection. In this way, MHV infection triggers axonal loss and macrophagemediated demyelination. However, we also observed in NDM strain infection, that axonal degeneration and myelin disruption can occur in the absence of macrophages. Therefore, MHV infection can both directly and indirectly cause axonal loss and demyelination. It may be that the failure of NDM strain to trigger macrophage-mediated damage is entirely a function of transport failure to the white matter.

The current studies further demonstrate that the molecular mechanisms underlying axonal damage are mediated by the spike glycoprotein. The recombinant DM and NDM viruses used are isogenic on a background of the DM MHV-A59 strain, and differ only by the virus-host attachment spike glycoprotein. In vivo, the spike protein host receptor is CEA (carcinoembryonic antigen), which functions as an intercellular adhesion molecule (Dveksler et al., 1993; Chen et al., 1997; Tan et al., 2002). However, there is evidence that other viral entry host factors may also be permissive for viral infection (Gallagher et al., 1992). Evidently, the difference in the spike proteins between the DM and NDM strains does not impair host-receptor interactions or viral entry since both strains effectively cause encephalitis following transcranial inoculation. The spike protein does, however, appear to play a critical role in axonal transport, as we found a lack of viral antigen spread 
and subsequent inflammation extending into spinal cord white matter following infection with the NDM strain in contrast to extensive white matter involvement secondary to DM strain infection. MHV spike is synthesized as an $180 \mathrm{kDa}$ glycosylated precursor that is posttranslationally cleaved into two $90 \mathrm{kDa}$ subunits, S1 and S2 (Frana et al., 1985), with a receptor binding domain in the S1 subunit (Kubo et al., 1994) that is responsible for the initial attachment of MHV to cell surface receptors. This binding event triggers a conformational change in spike that allows S2 to initiate fusion of the virus and host membranes (Gallagher and Buchmeier, 2001; Matsuyama and Taguchi, 2002; Zelus et al., 2003). A candidate fusion peptide domain has been identified within S2 (Luo and Weiss, 1998); however, the actual fusion peptide for the MHV spike has not been definitively identified. MHV-A59 and MHV-2 spike proteins have $82 \%$ amino acid sequence identity and $94 \%$ similarity, with the S2 domain relatively more conserved and S1 more variable (Spaan et al., 1988). They also differ in their cleavage signal site whereby MHV-A59 spike is cleaved posttranslationally into S1 and S2 subunits, but MHV-2 spike protein is not cleaved and unable to cause fusion in vivo and in vitro (Yamada et al., 1997). Thus, variable regions of the S1 domain or differences in the cleavage signal site between DM and NDM strains are candidate domains that may potentially explain the differential axonal transport and demyelination observed in our studies and will be evaluated further in future studies.

Together, our results demonstrate that the mechanisms of white matter injury in this model of CNS demyelinating disease are due to a combination of both axonal injury/loss and myelin damage. In DM infection, there is concomitant axonal loss and demyelination, and at least some demyelination is due to macrophage-mediated myelin stripping. One can hypothesize that axonal degeneration follows in this immune-mediated pathogenesis. Partial axonal loss also appears to occur secondary to direct viral-induced cytopathic effects, a non-immunemediated mechanism. Following NDM infection, although relatively rare, myelinated fibers in the spinal cord white matter exhibit early axonal degeneration in the absence of any associated inflammation. Therefore, MHV infection exhibits diverse mechanisms of axonal loss and demyelination that are both immune- and non-immune-mediated.

While the underlying mechanisms leading to the development of MS in patients is not known, numerous studies have provided evidence to suggest that a viral infection may trigger this autoimmune demyelinating disease (Hernán et al., 2001; Hemmer et al., 2002). The critical role of the spike glycoprotein in viral transport and subsequent axonal and myelin damage demonstrated in the current studies suggests that targeted disruption of the spike glycoprotein-host interaction has the potential to prevent the onset or progression of demyelination. Future experiments will be geared toward identifying how the spike proteins interact with the axonal transport system. Clues may be provided by other human viral neurologic infections, including those caused by such diverse viruses as herpes simplex and rabies, as they take advantage of the axonal transport system to maximize spread and neurovirulence. Our experimental mouse model provides an excellent means by which the particular host-virus interactions responsible for successful axonal transport can be dissected and clarified.

\section{References}

An SF, Giometto B, Groves M, Miller RF, Beckett AA, Gray F, Tavolato B, Scaravilli F (1997) Axonal damage revealed by accumulation of beta-APP in HIV-positive individuals without AIDS. J Neuropathol Exp Neurol 56:1262-1268.

Bjartmar C, Kinkel RP, Kidd G, Rudick RA, Trapp BD (2001) Axonal loss in normal-appearing white matter in a patient with acute MS. Neurology $57: 1248-1252$.

Cavanaugh D (1995) The coronavirus surface glycoprotein. In: The coronaviridae (Siddell SG, ed), pp 73-113. New York: Plenum.

Chen DS, Asanaka M, Chen FS, Shively JE, Lai MM (1997) Human carcinoembryonic antigen and biliary glycoprotein can serve as mouse hepatitis virus receptors. J Virol 71:1688-1691.

Czasch S, Paul S, Baumgärtner W (2006) A comparison of immunohistochemical and silver staining methods for the detection of diffuse plaques in the aged canine brain. Neurobiol Aging 27:293-305.

Dandekar AA, Wu GF, Pewe L, Perlman S (2001) Axonal damage is T cell mediated and occurs concomitantly with demyelination in mice infected with a neurotropic coronavirus. J Virol 75:6115-6120.

Das Sarma J, Fu L, Tsai JC, Weiss SR, Lavi E (2000) Demyelination determinants map to the spike glycoprotein gene of coronavirus mouse hepatitis virus. J Virol 74:9206-9213.

Das Sarma J, Scheen E, Seo SH, Koval M, Weiss SR (2002) Enhanced green fluorescent protein expression may be used to monitor murine coronavirus spread in vitro and in the mouse central nervous system. J Neurovirol 8:381-391.

Das Sarma J, Iacono K, Gard L, Marek R, Kenyon LC, Koval M, Weiss SR (2008) Demyelinating and nondemyelinating strains of mouse hepatitis virus differ in their neural cell tropism. J Virol 82:5519-5526.

Dveksler GS, Dieffenbach CW, Cardellichio CB, McCuaig K, Pensiero MN, Jiang GS, Beauchemin N, Holmes KV (1993) Several members of the mouse carcinoembryonic antigen-related glycoprotein family are functional receptors for the coronavirus mouse hepatitis virus-A59. J Virol $67: 1-8$.

Ferguson B, Matyszak MK, Esiri MM, Perry VH (1997) Axonal damage in acute multiple sclerosis lesions. Brain 120:393-399.

Frana MF, Behnke JN, Sturman LS, Holmes KV (1985) Proteolytic cleavage of the E2 glycoprotein of murine coronavirus: host-dependent differences in proteolytic cleavage and cell fusion. J Virol 56:912-920.

Gallagher TM, Buchmeier MJ (2001) Coronavirus spike proteins in viral entry and pathogenesis. Virology 279:371-374.

Gallagher TM, Buchmeier MJ, Perlman S (1992) Cell receptor-independent infection by a neurotropic murine coronavirus. Virology 191:517-522.

Hemmer B, Archelos JJ, Hartung HP (2002) New concepts in the immunopathogenesis of multiple sclerosis. Nat Rev Neurosci 3:291-301.

Hernán MA, Zhang SM, Lipworth L, Olek MJ, Ascherio A (2001) Multiple sclerosis and age at infection with common viruses. Epidemiology 12:301-306.

Iwasaki Y (1990) Pathology of chronic myelopathy associated with HTLV-I infection (HAM/TSP). J Neurol Sci 96:103-123.

Kornek B, Storch MK, Weissert R, Wallstroem E, Stefferl A, Olsson T, Linington C, Schmidbauer M, Lassmann H (2000) Multiple sclerosis and chronic autoimmune encephalomyelitis: a comparative quantitative study of axonal injury in active, inactive, and remyelinated lesions. Am J Pathol 157:267-276.

Kristensson K (1996) Sorting signals and targeting of infectious agents through axons: an annotation to the 100 years' birth of the name "axon." Brain Res Bull 41:327-333.

Kubo H, Yamada YK, Taguchi F (1994) Localization of neutralizing epitopes and the receptor-binding site with the amino-terminal 330 amino acids of the murine coronavirus spike protein. J Virol 68:5403-5410.

Lavi E, Gilden DH, Wroblewska Z, Rorke LB, Weiss SR (1984) Experimental demyelination produced by the A59 strain of mouse hepatitis virus. Neurology 34:597-603.

Lovas G, Szilágyi N, Majtényi K, Palkovits M, Komoly S (2000) Axonal changes in chronic demyelinated cervical spinal cord plaques. Brain 123:308-317.

Lucchinetti C, Brück W, Noseworthy J (2001) Multiple sclerosis: recent developments in neuropathology, pathogenesis, magnetic resonance imaging studies and treatment. Curr Opin Neurol 14:259-269.

Luo Z, Weiss SR (1998) Roles in cell-to-cell fusion of two conserved hydrophobic regions in the murine coronavirus spike protein. Virology 244:483-494. 
Martin JR (1984) Intra-axonal virus in demyelinative lesions of experimental herpes simplex type 2 infection. J Neurol Sci 63:63-74.

Matsuyama S, Taguchi F (2002) Receptor-induced conformational changes of murine coronavirus spike protein. J Virol 76:11819-11826.

Mori I, Kimura Y (2001) Neuropathogenesis of influenza virus infection in mice. Microbes Infect 3:475-479.

Navas S, Seo SH, Chua MM, Das Sarma J, Lavi E, Hingley ST, Weiss SR (2001) Murine coronavirus spike protein determines the ability of the virus to replicate in the liver and cause hepatitis. J Virol 75:2452-2457.

Pasick JM, Kalicharran K, Dales S (1994) Distribution and trafficking of JHM coronavirus structural proteins and virions in primary neurons and the OBL-21 neuronal cell line. J Virol 68:2915-2928.

Raja F, Sherriff FE, Morris CS, Bridges LR, Esiri MM (1997) Cerebral white matter damage in HIV infection demonstrated using beta-amyloid precursor protein immunoreactivity. Acta Neuropathol 93:184-189.

Rall GF, Manchester M, Daniels LR, Callahan EM, Belman AR, Oldstone MB (1997) A transgenic mouse model for measles virus infection of the brain. Proc Natl Acad Sci U S A 94:4659-4663.

Sharma R, Narayana PA, Wolinsky JS (2001) Grey matter abnormalities in multiple sclerosis: proton magnetic resonance spectroscopic imaging. Mult Scler 7:221-226

Shindler KS, Kenyon LC, Dutt M, Hingley ST, Das Sarma J (2008) Experimental optic neuritis induced by a demyelinating strain of mouse hepatitis virus. J Virol 82:8882-8886.
Spaan W, Cavanagh D, Horzinek MC (1988) Coronaviruses. Structure and genome expression. J Gen Virol 69:2939-2952.

Tan K, Zelus BD, Meijers R, Liu JH, Bergelson JM, Duke N, Zhang R, Joachimiak A, Holmes KV, Wang JH (2002) Crystal structure of murine sCEACAM1a[1,4]: a coronavirus receptor in the CEA family. EMBO J 21:2076-2086.

Trapp BD, Peterson J, Ransohoff RM, Rudick R, Mörk S, Bö L (1998) Axonal transection in the lesions of multiple sclerosis. N Engl J Med 338:278-285.

Trapp BD, Ransohoff R, Rudick R (1999) Axonal pathology in multiple sclerosis: relationship to neurologic disability. Curr Opin Neurol 12: 295-302.

Tsunoda I, Fujinami RS (2002) Inside-out versus outside-in models for virus induced demyelination: axonal damage triggering demyelination. Springer Semin Immunopathol 24:105-125.

Tsunoda I, Kuang LQ, Libbey JE, Fujinami RS (2003) Axonal injury heralds virus-induced demyelination. Am J Pathol 162:1259-1269.

Yamada YK, Takimoto K, Yabe M, Taguchi F (1997) Acquired fusion activity of a murine coronavirus MHV-2 variant with mutations in the proteolytic cleavage site and the signal sequence of the S protein. Virology 227:215-219.

Zelus BD, Schickli JH, Blau DM, Weiss SR, Holmes KV (2003) Conformational changes in the spike glycoprotein of murine coronavirus are induced at $37^{\circ} \mathrm{C}$ by soluble murine CEACAM1 receptor glycoproteins or by pH 8. J Virol 77:830-840. 